\title{
Tuning of the electronic and optical properties of single-layer black phosphorus by strain
}

\author{
Deniz Çakır, ${ }^{*}$ Hasan Sahin, ${ }^{\dagger}$ and François M. Peeters ${ }^{\ddagger}$ \\ Department of Physics, University of Antwerp, Groenenborgerlaan 171, 2020 Antwerpen, Belgium \\ (Received 26 September 2014; revised manuscript received 4 November 2014; published 17 November 2014)

\begin{abstract}
Using first principles calculations we showed that the electronic and optical properties of single-layer black phosphorus (BP) depend strongly on the applied strain. Due to the strong anisotropic atomic structure of BP, its electronic conductivity and optical response are sensitive to the magnitude and the orientation of the applied strain. We found that the inclusion of many body effects is essential for the correct description of the electronic properties of monolayer BP; for example, while the electronic gap of strainless $\mathrm{BP}$ is found to be $0.90 \mathrm{eV}$ by using semilocal functionals, it becomes $2.31 \mathrm{eV}$ when many-body effects are taken into account within the $G_{0} W_{0}$ scheme. Applied tensile strain was shown to significantly enhance electron transport along zigzag direction of BP. Furthermore, biaxial strain is able to tune the optical band gap of monolayer BP from $0.38 \mathrm{eV}$ (at $-8 \%$ strain) to $2.07 \mathrm{eV}$ (at 5.5\%). The exciton binding energy is also sensitive to the magnitude of the applied strain. It is found to be $0.40 \mathrm{eV}$ for compressive biaxial strain of $-8 \%$, and it becomes $0.83 \mathrm{eV}$ for tensile strain of $4 \%$. Our calculations demonstrate that the optical response of BP can be significantly tuned using strain engineering which appears as a promising way to design novel photovoltaic devices that capture a broad range of solar spectrum.
\end{abstract}

DOI: 10.1103/PhysRevB.90.205421

PACS number(s): 73.22.-f, 73.63.-b, 78.67.-n

\section{INTRODUCTION}

With the synthesis of single graphene [1-3] layers, a new era of two-dimensional (2D) monolayer materials emerged in condensed matter physics. It is believed that further advances in synthesis and fabrication of those monolayer crystals will pave the way in the exploration of many novel materials with exotic functionalities. Nowadays, single layers of functionalized graphenes [4-10], transition metal dichalcogenides (TMDs) [11-18], and boron nitride (BN) [19] are readily accessible and some applications in nanoscale devices have been demonstrated. Although graphene is a fascinating $2 \mathrm{D}$ material, the lack of a band gap in its electronic spectrum leads to a search for similar ultrathin materials having a nonzero band gap.

Recently, the successful synthesis of single-layer phosphorus crystal "phosphorene" [so-called black phosphorus (BP)] triggered interest in this material [20-23]. Single-layer BP is an appealing material that can be implemented into various electronic device applications including gas sensor [24], p-n junction [25], solar cell application [26], and field effect transistor (FET) due to its sizable band gap $(\sim 0.9 \mathrm{eV})$ and higher carrier mobility as compared to $\mathrm{MoS}_{2}$ [20,21,27-30]. Li et al. [20] fabricated FETs based on few-layer black phosphorus crystals and achieved reliable transistor performance at room temperature. Moreover, the stability and anisotropic structural properties of monolayer phosphorene were predicted by Liu et al. [21]. They observed a high on-current, a high hole field-effect mobility, and a high on/off ratio in fewlayer phosphorene FETs. Buscema et al. [27] demonstrated that black phosphorus is an appealing candidate for tunable photodetection applications due its unique properties such as (i) FETs allowing for ambipolar operation in the dark state and (ii) broadband (from the visible region up to $940 \mathrm{~nm}$ ) with

\footnotetext{
*deniz.cakir@uantwerpen.be

†hasan.sahin@uantwerpen.be

†francois.peeters@uantwerpen.be
}

fast detection (rise time of about $1 \mathrm{~ms}$ ) when illuminated. In a recent theoretical study by Dai et al. [31] it was shown that the direct band gap of phosphorene depends on the number of layer (from 0.3 to $1.5 \mathrm{eV}$ ) and that a vertical electric field can be used to tune this band gap.

Recently, accurate tight-binding (and $G W$ ) description of the electronic band structure of one to four layers black phosphorus and the importance of the interlayer hoppings were reported [32]. In addition, phosphorene nanoribbons (PNRs) were investigated using density functional theory [33,34]. Tran et al. [33] reported the electronic structure and optical absorption spectra of monolayer phosphorene nanoribbons. They showed that the band gaps of armchair ribbons scale as $1 / W^{2}$, while zigzag PNRs exhibit a $1 / W$ behavior, where $W$ is the width of the nanoribbon. The direction-dependent width dependence of the band gap was attributed to the nonrelativistic behavior of electrons and holes along the zigzag direction and the relativistic behavior along the armchair direction. The respective PNRs' host electrons and holes with markedly different effective masses and optical absorption spectra were found to be suitable for a wide range of applications. Han et al. [34] showed that electronic properties (i.e., effective mass of charge carriers) of passivated phosphorene nanoribbons exhibit a strong dependence on the orientation and strain.

Although the electronic and structural properties of monoand few-layer phosphorenes have been investigated previously, the role of the strain on its properties have remained an open question so far. Recently, BP was shown to have a negative Poisson's ratio [35] and superior mechanical flexibility [36] that allows us to use it under extreme mechanical conditions. By using density functional theory and tight-binding models Rodin et al. [37] showed that the deformation in the direction normal to the crystal plane can be used to change the size of the energy band gap and induce a semiconductor-metal transition. In addition, Fei et al. [38] reported that the anisotropic freecarrier mobility of phosphorene can be controlled by applying biaxial and uniaxial strain. In the present study we investigate how its electronic and optical properties change under biaxial strain and calculate the exciton binding energy. We organize 


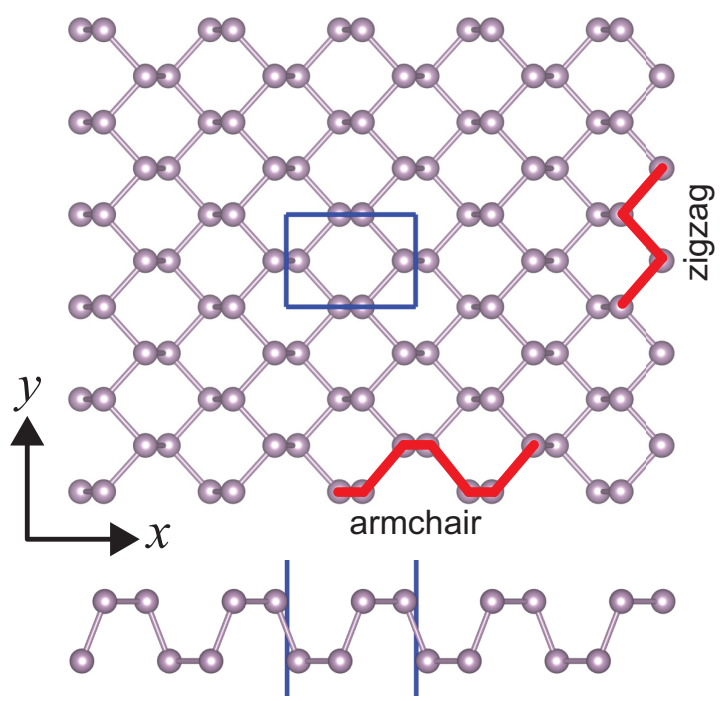

FIG. 1. (Color online) Top and side views of black phosphorus (BP) monolayer. The unit cell of BP is shown by the blue rectangle.

the paper as follows: electronic and transport properties of phosphorene under strain is presented in Sec. II and the optical response of monolayer phosphorene in the presence of biaxial strain is investigated in Sec. III. Our results are summarized in Sec. IV.

\section{TRANSPORT PROPERTIES}

Electronic transport through single-layer phosphorene is calculated by using the self-consistent nonequilibrium Green's functions (NEGF) technique as implemented in TRANSIESTA [39], which is interfaced with the SIESTA code [40]. A double- $\zeta$ (plus polarization) numerical orbital basis set is used for the $\mathrm{P}$ atom. We employ a Troullier-Martins norm-conserving pseudopotential [41], the GGA/PBE functional [42], and an energy cutoff for the real-space mesh of 200 Ry. The electron transport is calculated along the armchair $(x)$ and zigzag $(y)$ directions (see Fig. 1). In order to get accurate transmission spectrum, the 2D Brillouin zone normal to the transport direction is sampled by meshes composed of $100 \mathbf{k}$ points in the periodic direction.

Figure 2 shows the evolution of the band structure of the single-layer BP with the applied in-plane biaxial tensile and compressive strain $\left(\varepsilon_{x y}\right)$. Strain-free single-layer BP is a direct band gap semiconductor with a GGA/PBE band gap of $0.9 \mathrm{eV}$ at the $\Gamma$ point. It is noteworthy to mention that BP has a highly anisotropic band dispersion around the band gap. In other words, the top of the valence band and the bottom of the conduction band have a much larger dispersion along the $\Gamma-X$ direction as compared to the rather flat bands along the $\Gamma-Y$ direction, resulting in significantly anisotropic electronic properties. For instance, it was recently shown that the effective mass of electrons and holes are highly anisotropic [38]. For a better insight, in Fig. 3, the real-space wave functions corresponding to the top of the valence band (marked as A), bottom of the conduction band (B), and the second lowest energy conduction band $(\mathrm{C})$ at the $\Gamma$ point are depicted for strainless BP. As seen in Fig. 3, these three bands

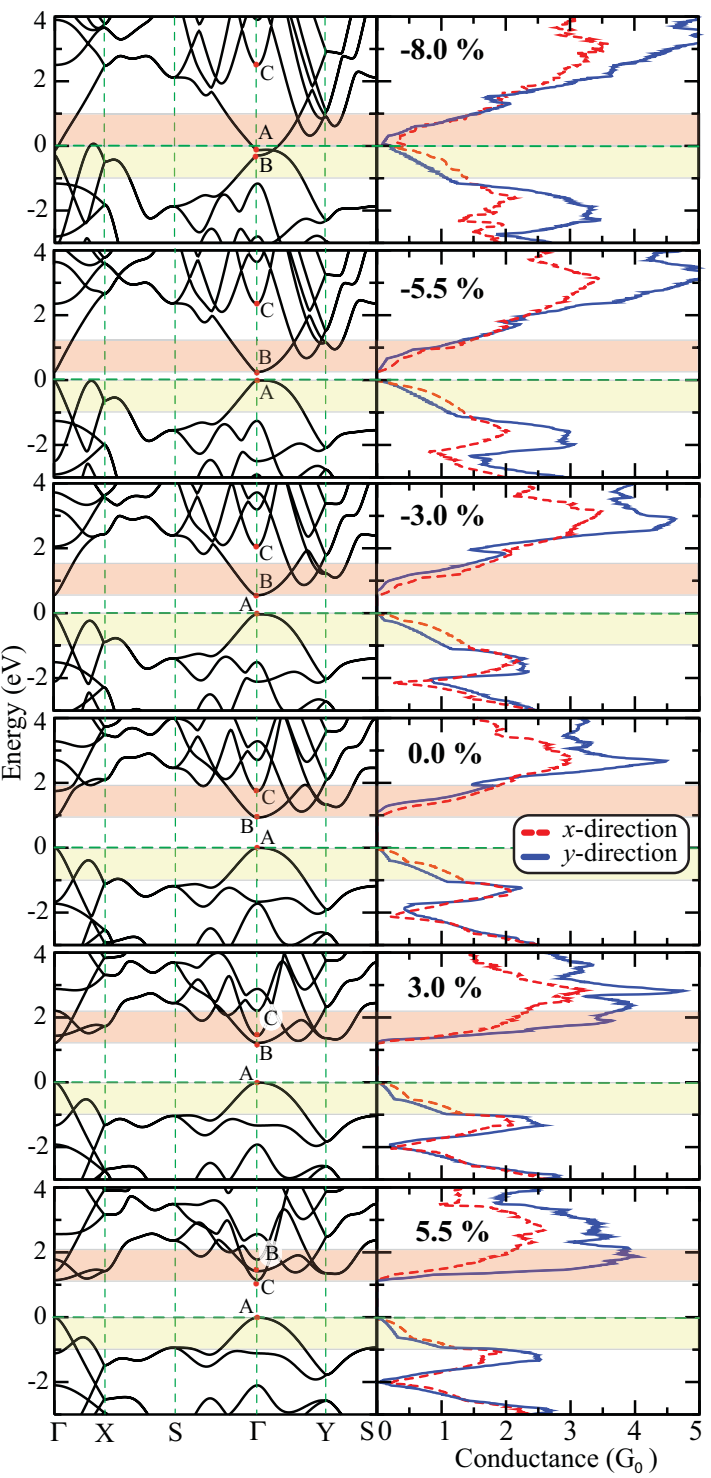

FIG. 2. (Color online) Band structure and conductance for single-layer BP for different strain values. A, B, and C mark the top of the valence band, bottom of the conduction band, and the second lowest conduction band at the $\Gamma$ point, respectively. Shaded regions depict the upper part of the valence and lower part of the conduction bands. Here the quantum of conductance is $G_{0}=\frac{2 e^{2}}{h}$.

display very different spatial characters. The valence band edge (point A) has a nonbonding character in the $y$ and an antibonding character in the $x$ direction. The conduction band edge (point B), which is dominated by the $p_{z}$ orbital, exhibits a bonding character along the $y$ direction. In contrast, it has a nonbonding nature in the $x$ direction.

For strain values of $-5.5 \% \leqslant \varepsilon_{x y} \leqslant+5.5 \%$, BP monolayer remains a direct band gap semiconductor at the $\Gamma$ point. At $\varepsilon_{x y}=-5.5 \%$, direct and indirect band gap values are very close. Although not shown in Fig. 2, when strain is $-6 \%$, BP becomes an indirect band gap semiconductor and a semiconductor-to-metal transition is observed above $-6.5 \%$. Interestingly, for uniaxial strains (either along zigzag or armchair directions), direct-to-indirect and 

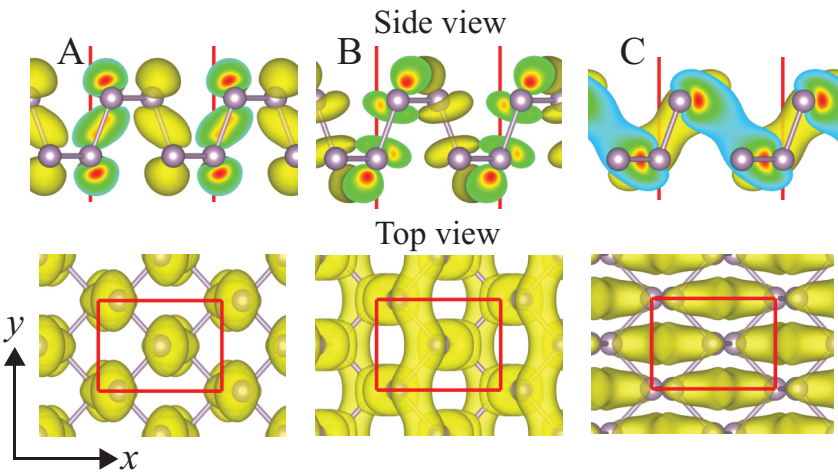

FIG. 3. (Color online) 3D band decomposed charge densities of band edges which are labeled as A, B, and C in Fig. 2 for strainless BP.

semiconductor-to-metal transitions occur at much larger strain values [43]. The band gap of BP exhibits a strong strain dependence. While compressive biaxial strain gives rise to a band gap lowering, tensile strain opens the band gap up to $\varepsilon_{x y}=4 \%$, but then reduces it at $\varepsilon_{x y}=5.5 \%$. The reason for lowering in the band gap under larger tensile strain is that while the band edge $\mathrm{B}$ moves away from the band edge $\mathrm{A}$, the edge $\mathrm{C}$ has the opposite tendency. Up to a critical tensile strain value at which the energy of the band edge $\mathrm{C}$ becomes lower than that of the band edge B, the band gap increases with strain. Once tensile strain exceeds the critical value, the band gap starts to decrease. For instance, while the band gap (at GGA-PBE level) is found to be $0.25 \mathrm{eV}$ at $\varepsilon_{x y}=-5.5 \%$, it becomes $1.19 \mathrm{eV}$ at $\varepsilon_{x y}=4 \%$ and drops down to $1.03 \mathrm{eV}$ at $\varepsilon_{x y}=5.5 \%$. As seen in Fig. 2, at $\varepsilon_{x y}=-8 \%$, A (B) point moves above (below) the Fermi level and BP is a metal for this strain value.

One would expect that the observed variation in the electronic structure under strain can translate into a modulation in the electron conduction. The right column of Fig. 2 shows the conductance of BP monolayer as a function of strain. Consistent with the band structure plots, the band gap values (within $-5.5 \% \leqslant \varepsilon_{x y} \leqslant 4 \%$ ) calculated in transport calculations decreases (increases) with compressive (tensile) strain. Due to the highly anisotropic electronic properties, conductance of monolayer BP shown in Fig. 2 exhibits a strong direction and strain dependence. In contrast, for $\mathrm{MoS}_{2}$ monolayer, it was found that the electrical conductivity does not depend on the direction of the current in the presence of isotropic strain [44]. Applying external strain switches the band ordering at the $\Gamma$ point of the conduction band; see Fig. 2.

As denoted in Fig. 3, since the band edge B has a bonding (nonbonding) character along the $y(x)$ direction, the expansion of BP monolayer (especially along $y$ direction) lowers the binding energy of this band, leading to an upward shift. In contrast, the band edge $\mathrm{C}$ has an antibonding character along the $x$ direction, and thus applying tensile strain results in a downward shift. However, compressive strain shifts the band edge $\mathrm{C}$ towards the higher energies to prevent the population of this band. For tensile strain, the conductivity along the $y$ direction around the conduction band edge significantly enhances as a result of strain induced rotation of the electronic conduction around the $\Gamma$ point. This can be clearly seen in Fig. 2 when comparing the evolution of the conductance with strain within the shaded region in the conduction band. The second lowest empty band (which is labeled as $\mathrm{C}$ in Fig. 2) moves down with increasing strain, whose effect can be directly seen in the electrical conduction along the $y$ direction. At $\varepsilon_{x y}=5.5 \%$, this band has lower energy than band B which is the lowest energy empty band for $-5.5 \% \leqslant \varepsilon_{x y}<4 \%$. In the vicinity of band edge $\mathrm{C}$, the effective mass of the charge carriers in the $y$ direction $(\Gamma-Y)$ is much smaller than that in the $x$ direction $(\Gamma-X)[38]$. In contrast, the effective mass of the charge carriers in the conduction band minimum (B point) exhibits the opposite behavior.

Figure 4 displays contour plots of the valence and conduction bands around the $\Gamma$ point, which illustrates the anisotropic nature of those bands. The change of band order at $\Gamma$ can be easily followed in Fig. 4.

Another important point is that the high value conductance peaks also move towards lower energies with increasing strain, which significantly increases the conductivity around the conduction band edge. Especially, the effect of strain on the different bands are more pronounced around the $\Gamma$ point. As seen in Fig. 2, the valence band along the $\Gamma-Y$ direction is flat, resulting in a very large effective mass for holes. Therefore, the conductivity is quite low for this band along the $\Gamma-Y$ direction (i.e., zigzag direction); see Fig. 2. In addition, the valence band is found to be insensitive to the applied strain, which results in a quite similar conductivity for all strain values considered in this study. It appears that tensile strain dramatically modifies the electron transport properties of single-layer BP.

\section{OPTICAL PROPERTIES}

One could expect that the excitonic effects are influential on the optical properties of BP due to the weak screening and reduced dimensionality. For a correct description of the optical properties of BP, many body interactions (electronelectron and electron-hole interaction, for instance) must be taken into account. In this work, we calculated the optical spectra of monolayer BP under both compressive and tensile biaxial strains $\left(\varepsilon_{x y}\right)$ using the Bethe-Salpeter-equation (BSE) method $[45,46]$ within the Vienna ab initio simulation package (VASP) [47,48]. First, hybrid-DFT calculations were performed within the HSE06 [49-51] approach for single-layer $\mathrm{BP}$ structure that was optimized using GGA-PBE. This is followed by one-shot $G W$ (i.e., $G_{0} W_{0}$ ) calculations to obtain the quasiparticle excitations [52-57]. Finally, we carried out BSE calculations on top of $G_{0} W_{0}$ in order to obtain the optical adsorption spectra by including excitonic effects using the Tamm-Dancoff approximation [58].

The BSE calculations were performed on a $9 \times 13 \times 1 k$ mesh within the Monkhorst-Pack scheme [59]. The energy cutoff for the wave functions and for the response functions were set to $400 \mathrm{eV}$ and $200 \mathrm{eV}$, respectively. Since the number of empty bands significantly influences the relative position of the quasiparticle energy states, we tested the convergence for 112 and 326 empty bands. The calculated quasiparticle gap and exciton binding energy are then converged within $0.05 \mathrm{eV}$. The six highest occupied valence bands and six lowest unoccupied conduction bands were included as the basis for the excitonic states. Since $G W$ calculations require a sufficiently 


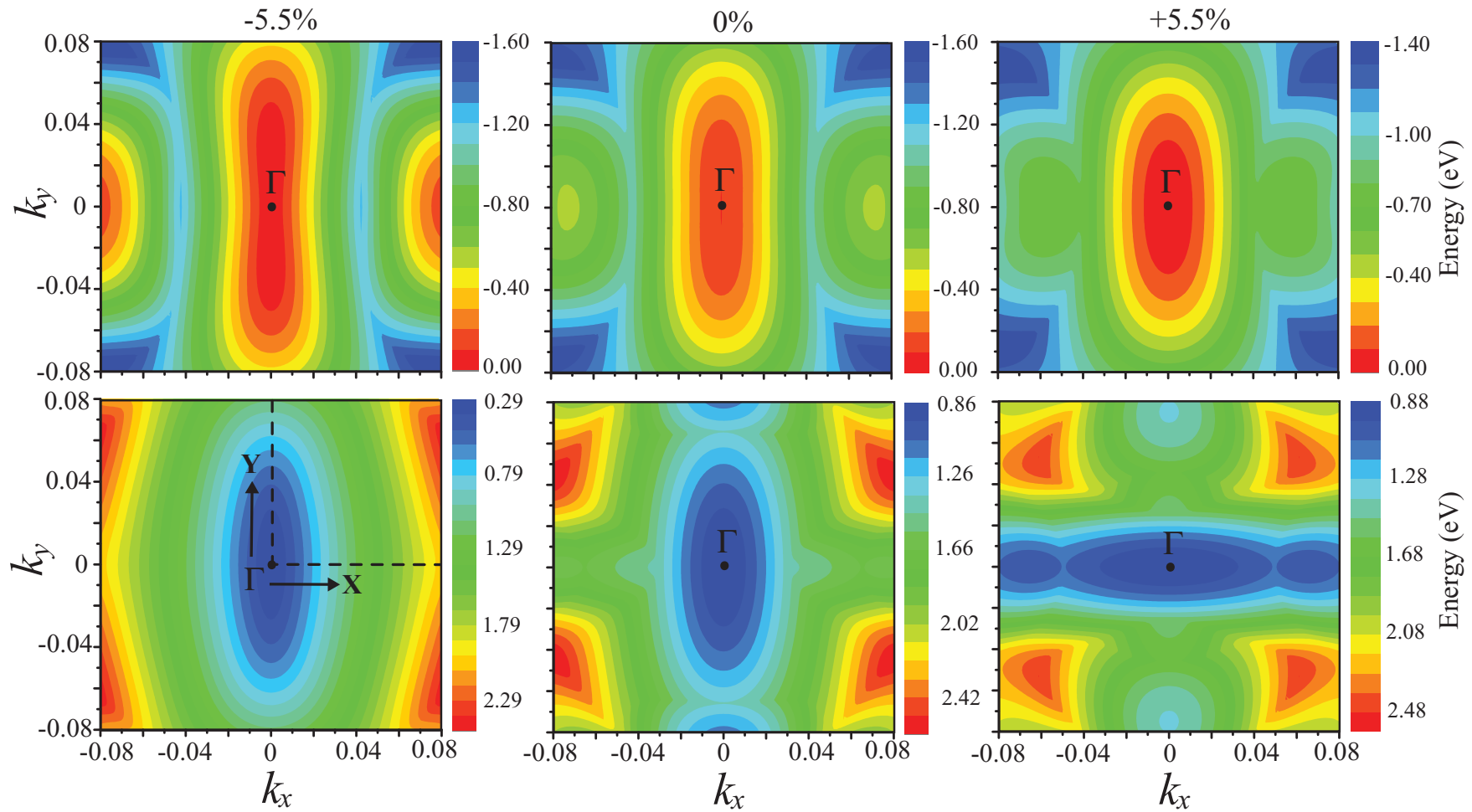

FIG. 4. (Color online) Contour plots of the valence (top) and the conduction (bottom) bands around the $\Gamma$ point for different strain values calculated with GGA-PBE functional.

large vacuum region, we use a vacuum region of at least $15 \AA$ to avoid spurious interaction between the periodic images. A complex shift of $\eta=0.05 \mathrm{eV}$ was employed to broaden the calculated absorption spectra.

Table I summarizes the calculated electronic gap ( $\left.E_{\text {gap }}\right)$, optical gap $\left(E_{\text {opt }}\right)$, and exciton binding energy $\left(E_{\text {exc }}=E_{\text {gap }}^{G_{0} W_{0}}-\right.$ $\left.E_{\text {opt }}\right)$ for different strain values. The variation of the electronic and the optical properties of BP as a function of biaxial strain is plotted in Fig. 5. As seen in Table I and Fig. 5, using hybrid functionals significantly enlarges $E_{\text {gap }}$. While $E_{\text {gap }}$ for strainless BP monolayer is calculated as $0.90 \mathrm{eV}$, consistent with previous calculations, in GGA-PBE it becomes $1.59 \mathrm{eV}$ when using HSE06. Inclusion of many body effects $\left(G_{0} W_{0}\right)$ further increases $E_{\text {gap }}$ to $2.31 \mathrm{eV}$ (see Table I). Previous theoretical studies predicted that $E_{\text {gap }}$ of single-layer BP

TABLE I. Calculated electronic gap ( $\left.E_{\text {gap }}\right)$, optical gap $\left(E_{\text {opt }}\right)$, and exciton binding energy $\left(E_{\text {exc }}\right)$ for different biaxial strain $\left(\varepsilon_{x y}\right)$ values in units of eV. Here, M stands for metallic behavior. Since BP is an indirect band gap semiconductor for the strain values of $-6 \%$ and $-8 \%$, we give the indirect band gap value. The direct gap is 0.02 $\mathrm{eV}$ bigger than the indirect one.

\begin{tabular}{|c|c|c|c|c|c|c|c|}
\hline & $\varepsilon_{x y}=$ & $-8 \%$ & $-6 \%$ & $-4 \%$ & 0 & $+4 \%$ & $+5.5 \%$ \\
\hline$E_{\text {gap }}^{P B E}$ & & M & 0.26 & 0.47 & 0.90 & 1.19 & 1.03 \\
\hline$E_{\text {gap }}^{H S E 06}$ & & 0.32 & 0.85 & 1.09 & 1.59 & 1.95 & 1.80 \\
\hline$E_{\mathrm{gap}}^{G_{0} W_{0}}$ & & 0.78 & 1.28 & 1.69 & 2.31 & 2.87 & 2.72 \\
\hline$E_{\mathrm{opt}}$ & & 0.38 & 0.75 & 1.07 & 1.61 & 2.04 & 2.07 \\
\hline$E_{\mathrm{exc}}$ & & 0.40 & 0.53 & 0.62 & 0.70 & 0.83 & 0.65 \\
\hline
\end{tabular}

increases (decreases) under tensile (compressive) strain [43]. We observe that not only $E_{\text {gap }}$ but also $E_{\text {opt }}$ and $E_{\text {exc }}$ are very sensitive to the applied strain. Therefore, by tuning strain, optical properties of BP can be easily modified. Adversely, $E_{\text {exc }}$ hardly changes under strain for $\mathrm{MoS}_{2}$ monolayer [60]. The experimental optical gap [21] for single-layer BP was shown to be around $1.45 \mathrm{eV}$, in fair agreement with the present calculated value of $1.61 \mathrm{eV}$. In a recent experiment,

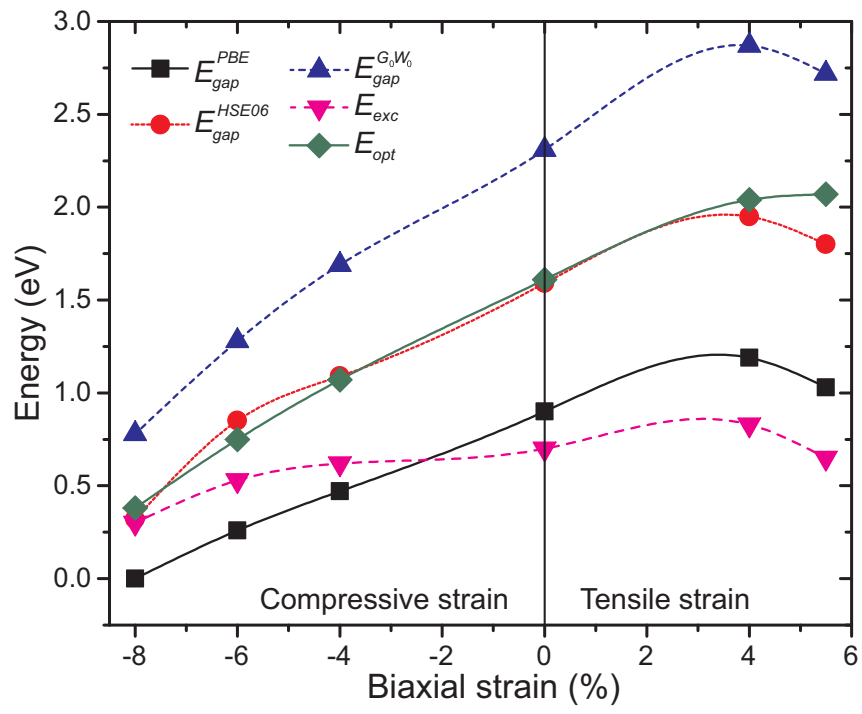

FIG. 5. (Color online) Electronic band gap $\left(E_{\text {gap }}\right)$, optical gap $\left(E_{\text {opt }}\right)$, and exciton binding energy $\left(E_{\text {exc }}\right)$ as a function of biaxial strain for different exchange-correlation functionals. 

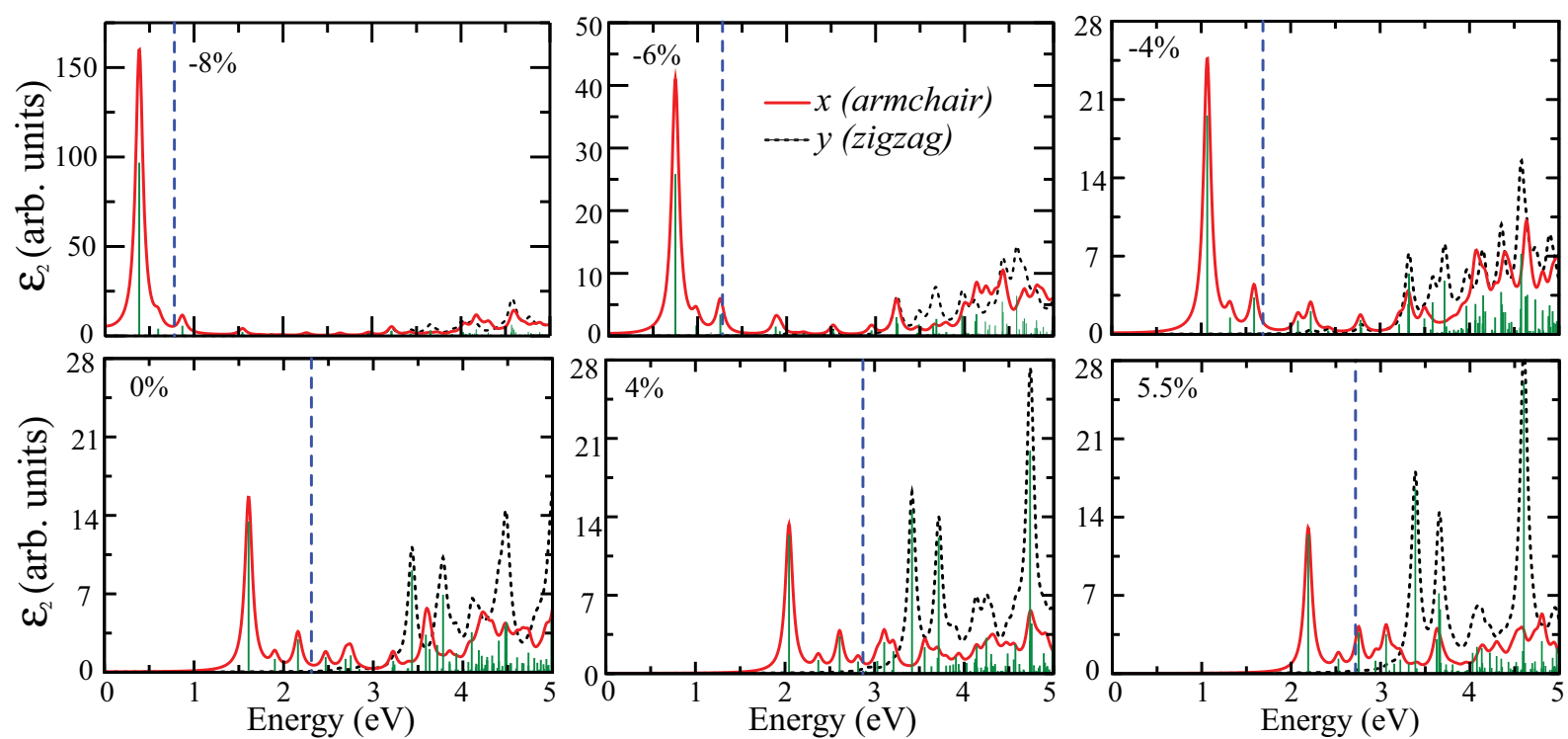

FIG. 6. (Color online) $G_{0} W_{0}+\mathrm{BSE}$ absorption spectra for monolayer BP as a function of strain. Blue vertical dashed lines mark the electronic band gap calculated at the level of $G_{0} W_{0}$. Green vertical lines represent the relative oscillator strengths for the optical transitions.

since $\mathrm{BP}$ is placed on a $\mathrm{SiO}_{2}$ surface, it is expected that screening effects weakens the binding and decreases the exciton binding energy, in qualitative agreement with our simulations [21]. Due to the reduced dimensionality and weak screening, our calculations predict a large exciton binding energy for strainless monolayer $\mathrm{BP}$ of $0.7 \mathrm{eV}$, which is in good agreement with recent theoretical works [61,62]. Note that the calculated exciton binding energy for BP is comparable to those found for other monolayer semiconductors such as $\mathrm{MoS}_{2} . E_{\text {exc }}$ has been found to be in the order of $1 \mathrm{eV}$ for single-layer $\mathrm{MoS}_{2}[60,63-66]$. Another point is that tensile strain up to $\varepsilon_{x y}=4 \%$ enhances the exciton binding due to the weakening of the dielectric screening as a result of a significant increase of the electronic band gap. It is worth mentioning that when one uses PBE wave functions and eigenvalues as inputs for the $G W$ calculations instead of HSE06, $E_{\mathrm{opt}}$ is found to be $1.30 \mathrm{eV}$ instead of $1.61 \mathrm{eV}$, pointing to the significance of the HSE step in calculating the quasiparticle and optical gap.

As mentioned in the previous section, GGA-PBE functional predicts that single-layer BP undergoes a semiconductor-tometal transition when $\varepsilon_{x y}=-8 \%$. However, both HSE06 and $G_{0} W_{0}$ predict that BP is an indirect band gap semiconductor at this strain value with a gap of $0.32(0.78) \mathrm{eV}$ with HSE06 $\left(G_{0} W_{0}\right)$. In line with the present study, it was previously shown that the calculated vertical compressive strain value that is needed to induce a semiconductor-to-metal transition in bilayer $\mathrm{MoS}_{2}$ was found to be much larger in $G W$ than that in GGA-PBE [67]. Therefore, to predict the transition point accurately, $G W$ calculations are essential.

Figure 6 displays the optical absorption spectra $\left(\varepsilon_{2}\right)$ of single-layer BP for light polarized along $x$ (armchair) and $y$ (zigzag) directions for different strain values. We also show the electronic band gap values calculated with $G_{0} W_{0}$. Similar to the transport properties, the optical absorption spectra displays a strong orientation dependence. It is clear that excitonic effects largely affect the optical spectra of BP.
For all strain values, we notice an absorption peak along the $x$ direction, since BP absorbs $x$-polarized light due to its anisotropic electronic structure. This peak is observed at $1.61 \mathrm{eV}$ for strain-free BP, and it moves to lower (higher) energy under compressive (tensile) strain. We find three bound exciton states when $\varepsilon_{x y}=-4 \%$ and $\varepsilon_{x y}=0$ and four bound exciton states for $\varepsilon_{x y}=4 \%$ with the wave function localized in the $x$ direction. Adversely, there is no bound exciton that is localized in the $y$ direction, meaning that BP is transparent to polarized light along the $y$ direction and when an exciton is formed, it strongly localizes along the $x$ direction. Increasing tensile strain from $4 \%$ to $5.5 \%$ decreases the electronic band gap (at the $G_{0} W_{0}$ level) and optical gap to 2.72 and $2.07 \mathrm{eV}$, respectively. Similarly, exciton binding weakens and $E_{\text {exc }}$ for $\varepsilon_{x y}=5.5 \%$ becomes very close to that for $\varepsilon_{x y}=-4 \%$. In addition, at $\varepsilon_{x y}=5.5 \%$, the number of bound exciton states drops from four to two. Interestingly, while $E_{\text {opt }}$ and $E_{\text {exc }}$ increase up to $\varepsilon_{x y}=4 \%$ and then decrease at $\varepsilon_{x y}=5.5 \%$, the first absorption peak always moves to higher energies as the strain changes from compressive to tensile.

These results confirm that strain has a significant effect on the optical properties. For instance, the absorption energy window of monolayer BP is tunable via strain. While BP monolayer under $-4 \%$ compressive strain absorbs infrared light (energies between $1.1 \mathrm{eV}$ and $1.7 \mathrm{eV}$ ), strainless BP can absorb the low energy part of the visible light spectrum (up to $2.4 \mathrm{eV}$ ). In addition, applying $4 \%$ tensile strain makes BP active almost over the whole visible range. Similar to interesting properties revealed by recent experiments on $\mathrm{MoS}_{2}$ [68], strain engineering can be used to tune the optical properties of photovoltaic devices made from a strain engineered BP monolayer.

\section{CONCLUSION}

In conclusion, we performed first-principles calculations in order to determine the effect of biaxial strain on the electronic 
transport and optical properties of BP. We find that the optical absorption spectrum and the carrier conductivity are highly anisotropic and strongly depend on the amount of applied strain. The large variation in the calculated electronic band gap of BP shows clearly the importance of many body effects in electronic structure calculations. The exciton binding is found to be large due to the reduced dimensionality and weak screening. $E_{\text {exc }}$ is $0.70 \mathrm{eV}$ for stainless BP, and it increases (decreases) to $0.83 \mathrm{eV}(0.40 \mathrm{eV})$ at the strain value of $+4 \%(-8 \%)$. In addition the optical gap varies by $1.5 \mathrm{eV}$ when the strain changes from compressive to tensile. Here, strain engineering appears as a quite exciting way to tune the optical response and the electrical conductivity of $\mathrm{BP}$, which is potentially useful for device applications including flexible electronics and optical devices due to its atomically thin crystal structure and direct band gap.

\section{ACKNOWLEDGMENTS}

This work was supported by the Flemish Science Foundation (FWO-Vl) and the Methusalem foundation of the Flemish government. Computational resources were provided by TUBITAK ULAKBIM, High Performance and Grid Computing Center (TR-Grid e-Infrastructure), and HPC infrastructure of the University of Antwerp (CalcUA) a division of the Flemish Supercomputer Center (VSC), which is funded by the Hercules foundation. H.S. is supported by a FWO Pegasus Marie Curie-long Fellowship. D.C. is supported by a FWO Pegasus-short Marie Curie Fellowship.
[1] K. S. Novoselov, A. K. Geim, S. V. Morozov, D. Jiang, Y. Zhang, S. V. Dubonos, I. V. Grigorieva, and A. A. Firsov, Science 306, 666 (2004).

[2] K. S. Novoselov, A. K. Geim, S. V. Morozov, D. Jiang, M. I. Katsnelson, I. V. Grigorieva, S. V. Dubonos, and A. A. Firsov, Nature (London) 438, 197 (2005).

[3] K. S. Novoselov, Z. Jiang, Y. Zhang, S. V. Morozov, H. L. Stormer, U. Zeitler, J. C. Maan, G. S. Boebinger, P. Kim, and A. K. Geim, Science 315, 1379 (2007).

[4] J. O. Sofo, A. S. Chaudhari, and G. D. Barber, Phys. Rev. B 75, 153401 (2007).

[5] H. Sahin, C. Ataca, and S. Ciraci, Appl. Phys. Lett. 95, 222510 (2009).

[6] R. R. Nair, W. Ren, R. Jalil, I. Riaz, V. G. Kravets, L. Britnell, P. Blake, F. Schedin, A. S. Mayorov, S. Yuan, M. I. Katsnelson, H. M. Cheng, W. Strupinski, L. G. Bulusheva, A. V. Okotrub, I. V. Grigorieva, A. N. Grigorenko, K. S. Novoselov, and A. K. Geim, Small 6, 2877 (2010).

[7] H. Sahin, M. Topsakal, and S. Ciraci, Phys. Rev. B 83, 115432 (2011).

[8] H. Peelaers, A. D. Hernandez-Nieves, O. Leenaerts, B. Partoens, and F. M. Peeters, Appl. Phys. Lett. 98, 051914 (2011).

[9] H. Sahin and S. Ciraci, J. Phys. Chem. C 116, 24075 (2012).

[10] A. L. Walter, H. Sahin, K.-J. Jeon, A. Bostwick, S. Horzum, R. Koch, F. Speck, M. Ostler, P. Nagel, M. Merz, S. Schupler, L. Moreschini, Y. J. Chang, T. Seyller, F. M. Peeters, K. Horn, and E. Rotenberg, ACS Nano 8, 7801 (2014).

[11] M. Chhowalla, H. S. Shin, G. Eda, L. J. Li, K. P. Loh, and H. Zhang, Nat. Chem. 5, 263 (2013).

[12] S. Tongay, H. Sahin, C. Ko, A. Luce, W. Fan, K. Liu, J. Zhou, Y. S. Huang, C. H. Ho, J. Yan, D. F. Ogletree, S. Aloni, J. Ji, S. Li, J. Li, F. M. Peeters, and J. Wu, Nat. Commun. 5, 3252 (2014).

[13] B. Sipos, A. F. Kusmartseva, A. Akrap, H. Berger, L. Forro, and E. Tutis, Nat. Mater. 7, 960 (2008).

[14] J. N. Coleman, M. Lotya, A. O’Neill, S. D. Bergin, P. J. King, U. Khan, K. Young, A. Gaucher, S. De, R. J. Smith, I. V. Shvets, S. K. Arora, J. J. Boland, J. J. Wang, J. F. Donegan, J. C. Grunlan, G. Moriarty, A. Shmeliov, R. J. Nicholls, J. M. Perkins, E. M. Grieveson, K. Theuwissen, D. W. McComb, P. D. Nellist, and V. Nicolosi, Science 331, 568 (2011).
[15] A. Splendiani, L. Sun, Y. Zhang, T. Li, J. Kim, C. Y. Chim, G. Galli, and F. Wang, Nano Lett. 10, 1271 (2010).

[16] Q. H. Wang, K. Kalantar-Zadeh, A. Kis, J. N. Coleman, and M. S. Strano, Nat. Nanotechnol. 7, 699 (2012).

[17] H. Sahin, S. Tongay, S. Horzum, W. Fan, J. Zhou, J. Li, J. Wu, and F. M. Peeters, Phys. Rev. B 87, 165409 (2013).

[18] D. Çakır, F. M. Peeters, and C. Sevik, Appl. Phys. Lett. 104, 203110 (2014).

[19] K. K. Kim, A. Hsu, X. Jia, S. M. Kim, Y. Shi, M. Hofmann, D. Nezich, J. F. Rodriguez-Nieva, M. Dresselhaus, T. Palacios, and J. Kong, Nano Lett. 12, 161 (2012).

[20] L. Li, Y. Yu, G. J. Ye, Q. Ge, X. Ou, H. Wu, D. Feng, X. H. Chen, and Y. Zhang, Nat. Nanotechnol. 9, 372 (2014).

[21] H. Liu, A. T. Neal, Z. Zhu, Z. Luo, X. Xu, D. Tomanek, and P. D. Ye, ACS Nano 8, 4033 (2014).

[22] E. S. Reich, Nature (London) 506, 19 (2014).

[23] H. Liu, Y. Du, Y. Deng, and P. D. Ye, Chem. Soc. Rev., doi:10.1039/C4CS00257A (2015).

[24] L. Kou, T. Frauenheim, and C. Chen, J. Phys. Chem. Lett. 5, 2675 (2014).

[25] M. Buscema, D. J. Groenendijk, G. A. Steele, H. S. J. van der Zant, and A. Castellanos-Gomez, Nat. Commun. 5, 4651 (2014).

[26] Y. Deng, Z. Luo, N. J. Conrad, H. Liu, Y. Gong, S. Najmaei, P. M. Ajayan, J. Lou, X. Xu, and P. D. Ye, ACS Nano 8, 8292 (2014).

[27] M. Buscema, D. J. Groenendijk, S. I. Blanter, G. A. Steele, H. S. J. van der Zant, and A. Castellanos-Gomez, Nano Lett. 14, 3347 (2014).

[28] J. Qiao, X. Kong, Z.-X. Hu, F. Yang, and W. Ji, Nat. Commun. 5, 4475 (2014).

[29] F. Xia, H. Wang, and Y. Jia, Nat. Commun. 5, 4458 (2014).

[30] A. Castellanos-Gomez, L. Vicarelli, E. Prada, J. O. Island, K. L. Narasimha-Acharya, S. I. Blanter, D. J. Groenendijk, M. Buscema, G. A. Steele, J. V. Alvarez, H. W. Zandbergen, J. J. Palacios, and H. S. J. van der Zant, 2D Mater. 1, 025001 (2014).

[31] J. Dai and X. C. Zeng, J. Phys. Chem. Lett. 5, 1289 (2014).

[32] A. N. Rudenko and M. I. Katsnelson, Phys. Rev. B 89, 201408(R) (2014).

[33] V. Tran and L. Yang, Phys. Rev. B 89, 245407 (2014).

[34] X. Han, H. M. Stewart, S. A. Shevlin, C. A. Catlow, and Z. X. Guo, Nano Lett. 14, 4607 (2014). 
[35] J.-W. Jiang and H. S. Park, Nat. Commun. 5, 4727 (2014).

[36] Q. Wei and X. Peng, Appl. Phys. Lett. 104, 251915 (2014).

[37] A. S. Rodin, A. Carvalho, and A. H. Castro Neto, Phys. Rev. Lett. 112, 176801 (2014).

[38] R. Fei and L. Yang, Nano Lett. 14, 2884 (2014).

[39] M. Brandbyge, J.-L. Mozos, P. Ordejón, J. Taylor, and K. Stokbro, Phys. Rev. B 65, 165401 (2002).

[40] J. M. Soler, E. Artacho, J. D. Gale, A. Garcia, J. Junquera, P. Ordejón, and D. Sánchez-Portal, J. Phys.: Condens. Matter. 14, 2745 (2002).

[41] N. Troullier and J. L. Martins, Phys. Rev. B 43, 1993 (1991).

[42] J. P. Perdew, K. Burke, and M. Ernzerhof, Phys. Rev. Lett. 77, 3865 (1996).

[43] X. Peng, Q. Wei, and A. Copple, Phys. Rev. B 90, 085402 (2014).

[44] M. Ghorbani-Asl, S. Borini, A. Kuc, and T. Heine, Phys. Rev. B 87, 235434 (2013).

[45] E. E. Salpeter and H. A. A. Bethe, Phys. Rev. 84, 1232 (1951).

[46] G. Onida, L. Reining, and A. Rubio, Rev. Mod. Phys. 74, 601 (2002).

[47] G. Kresse and J. Furthmuller, Comput. Mater. Sci. 6, 15 (1996).

[48] G. Kresse and J. Furthmüller, Phys. Rev. B 54, 11169 (1996).

[49] J. Heyd, G. E. Scuseria, and M. Ernzerhof, J. Chem. Phys. 118, 8207 (2003).

[50] J. Paier, M. Marsman, K. Hummer, G. Kresse, I. C. Gerber, and J. G. Ángyán, J. Chem. Phys. 125, 249901 (2006).

[51] J. Heyd, G. E. Scuseria, and M. Ernzerhof, J. Chem. Phys. 124, 219906 (2006).

[52] L. Hedin, Phys. Rev. 139, A796 (1965).
[53] M. S. Hybertsen and S. G. Louie, Phys. Rev. B 34, 5390 (1986).

[54] R. W. Godby, M. Schlüter, and L. J. Sham, Phys. Rev. B 37, 10159 (1988).

[55] M. Shishkin and G. Kresse, Phys. Rev. B 74, 035101 (2006).

[56] M. Shishkin and G. Kresse, Phys. Rev. B 75, 235102 (2007).

[57] M. Shishkin, M. Marsman, and G. Kresse, Phys. Rev. Lett. 99, 246403 (2007).

[58] J. Paier, M. Marsman, and G. Kresse, Phys. Rev. B 78, 121201(R) (2008).

[59] H. J. Monkhorst and J. D. Pack, Phys. Rev. B 13, 5188 (1976).

[60] H. Shi, H. Pan, Y.-W. Zhang, and B. I. Yakobson, Phys. Rev. B 87, 155304 (2013).

[61] V. Tran, R. Soklaski, Y. Liang, and L. Yang, Phys. Rev. B 89, 235319 (2014).

[62] A. S. Rodin, A. Carvalho, and A. H. Castro Neto, Phys. Rev. B 90, 075429 (2014).

[63] D. Y. Qiu, F. H. da Jornada, and S. G. Louie, Phys. Rev. Lett. 111, 216805 (2013).

[64] H.-P. Komsa and A. V. Krasheninnikov, Phys. Rev. B 86, 241201(R) (2012).

[65] H.-P. Komsa and A. V. Krasheninnikov, Phys. Rev. B 88, 085318 (2013).

[66] A. Ramasubramaniam, Phys. Rev. B 86, 115409 (2012).

[67] S. Bhattacharyya and A. K. Singh, Phys. Rev. B 86, 075454 (2012).

[68] J. Feng, X. Qian, C.-W. Huang, and J. Li, Nat. Photon. 6, 866 (2012). 